University of Wollongong

Research Online

Faculty of Engineering and Information

Faculty of Engineering and Information

Sciences - Papers: Part A

Sciences

$1-1-2016$

\title{
Analysis of laminated crack defect in the upsetting process of heavy disk- shaped forgings
}

Minting Wang

University of Wollongong, mwang@uow.edu.au

Dongying Li

Yanshan University

Fan Wang

Yanshan University

Xinliang Zang

Yanshan University

Xuetong Li

Yanshan University

See next page for additional authors

Follow this and additional works at: https://ro.uow.edu.au/eispapers

Part of the Engineering Commons, and the Science and Technology Studies Commons

Research Online is the open access institutional repository for the University of Wollongong. For further information contact the UOW Library: research-pubs@uow.edu.au 


\title{
Analysis of laminated crack defect in the upsetting process of heavy disk-shaped forgings
}

\begin{abstract}
Using nonlinear finite element method, a thermo-mechanical coupled simulation model for the formation mechanism of the laminated crack defect has been established in the upsetting of heavy disk-shaped forgings. Through numerical simulation, the distributions of stress, equivalent strain and strain rate were analysed. Meanwhile the distribution diagram of stress state evolution was obtained, and the uncoordinated deformation, under tri-lateral compression, is determined as the main reason leading to laminated crack defect. To reveal the characteristics of the uncoordinated deformation, the variations of each variable and its gradient in numerical simulation were presented, and a combined prediction model of laminated crack defect were proposed based on degree of deformation and gradient of deformation speed. Subsequently, the morphology and distribution of laminated crack were obtained in the centre of forging using the prediction model. Comparison of calculation results and experimental data indicates that both of them match well. In addition, the effect of friction coefficient on the deformation is also presented. The results show that the decreasing of friction coefficient is an effective measure to restrain the laminated crack defect.
\end{abstract}

\section{Keywords}

crack, upsetting, laminated, analysis, process, heavy, disk, forgings, defect, shaped

\section{Disciplines}

Engineering | Science and Technology Studies

\section{Publication Details}

Wang, M., Li, D., Wang, F., Zang, X., Li, X., Xiao, H., Du, F., Zhang, F. C. \& Jiang, Z. (2016). Analysis of laminated crack defect in the upsetting process of heavy disk-shaped forgings. Engineering Failure Analysis, 59 197-210.

\section{Authors}

Minting Wang, Dongying Li, Fan Wang, Xinliang Zang, Xuetong Li, Hong Xiao, Fengshan Du, F C. Zhang, and Zhengyi Jiang 


\title{
Analysis of Laminated Crack Defect in the Upsetting Process of Heavy Disk-shaped Forgings
}

\author{
Minting Wang ${ }^{1,2}, \mathrm{D} \mathrm{Li}^{1}$, Fan Wang ${ }^{1}$, Xinliang Zang ${ }^{1}$, Xuetong $\mathrm{Li}^{1}$, Hong Xiao ${ }^{1}$, Fengshan $\mathrm{Du}^{1}$, F.C. Zhang ${ }^{1}$, \\ Zhengyi Jiang ${ }^{2 *}$ \\ 1 College of Mechanical Engineering, Yanshan University, Qinhuangdao 066004, China \\ 2 School of Mechanical, Materials and Mechatronic Engineering, University of Wollongong, Wollongong, NSW 2522, Australia
}

\begin{abstract}
Using nonlinear finite element method, a coupling thermo-mechanical simulation model for the formation mechanism of the laminated crack defect has been established in the upsetting of heavy disk-shaped forgings. Through numerical simulation, the distributions of stress, equivalent strain and strain rate were analyzed. Meanwhile the distribution diagram of stress state evolution was obtained, and the uncoordinated deformation, under tri-lateral compression, is determined as the main reason leading to laminated crack defect. To reveal the characteristics of the uncoordinated deformation, the variations of each variable and its gradient in numerical simulation were presented, and a combined prediction model of laminated crack defect were proposed based on degree of deformation and gradient of deformation speed. Subsequently, the morphology and distribution of laminated crack were obtained in the centre of forging using the prediction model. Comparison of calculation results and experimental data indicates that both of them match well. In addition, the effect of friction coefficient on the deformation is also presented. The results show that the decreasing of friction coefficient is an effective measure to restrain the laminated crack defect.
\end{abstract}

Keywords: Heavy disk-shaped forgings; Laminated Crack; Deformation gradient; Finite element method

\section{Introduction}

Heavy disk-shaped forgings are commonly used for key parts of equipment in the nuclear, electronics, heating fields, such as tube plate and impeller in turbine boiler, top cover of pressure vessel for nuclear reactor, tube plate of heat exchanger, and bottom head of chemical containers etc. The forming of the disk-shaped forgings is carried out by forging, and mainly upsetting. In the upsetting process, the defects of the cast dendritic microstructure and shrinkage/porosity will be fixed by larger forging ratio in order to improve the quality of forgings. According to the theory of plasticity, if friction is ignored, the upsetting between flats can be simplified to be the single compression. However, the existing of friction leads to complex changes of the stress-strain state in the forgings, many problems cannot be solved, and even cannot be qualitatively analyzed ${ }^{[1]}$. Therefore, there were some misunderstandings on the upsetting of cylinder between flats in a long period of time, namely state of tri-lateral compressive stresses is always produced in the centre of deformation body, regardless the ratio of height to diameter (transient state) of the forging ${ }^{[2,3]}$. In response to this problem, Prof. Liu ${ }^{[3]}$ proposed two new theories, namely the tensile stress theory of rigid-plastic mechanics ${ }^{[4-6]}$ and the shear stress theory of hydrostatic stress mechanics ${ }^{[7,8]}$, which presented the evolution law of complex stress state in the forgings, and the qualitatively physical simulation experiments were also carried out ${ }^{[4]}$. Subsequently, the theories were verified by aid of the Generalized Slip-line Method ${ }^{[9]}$ and Mechanical Slab Method ${ }^{[10]}$.

Inner laminated crack during upsetting is serious forging defects of disk-shaped forgings, which reduces the qualified rate of ultrasonic inspection of forgings, and significantly affects the mechanical properties of materials and safety performance. Recent plastic mechanics theory cannot explain horizontal-type laminated crack defects. Many scholars presented different views on its formation mechanism, which include hydrogen flaking, inclusions and unclosed porosity under axial compression ${ }^{[11-13]}$. RST effect (Rigid Slid Tearing Effect) is also proposed ${ }^{[14,15]}$, that because the deformation energy needs to be released after two rigid areas contact, if

\footnotetext{
* Corresponding author. Tel.: +61 24221 4545; fax: +61 242215474.
}

E-mail address: jiang@uow.edu.au 
forced deformation under compressive stress continues, it will lead to laminated rigid slip deformation, and tearing failure occurs when reaching the material allowed strength of shear strain. Shear stress theory of hydrostatic stress mechanics proposed by $\mathrm{Liu}^{[7,8]}$, exposes mechanical behavior and deformation mechanism of the centre of the sample during upsetting when the ratio of height to diameter of forgings is less than 1. It is believed that the stress state in the stagnant deformation zone at the end of forging is hydrostatic pressure, where the shear stress can exceed critical shearing strength of material, and laminated shear crack parallel to the section appears where the shear deformation is very significant, namely reaching the critical condition. However, most investigations on heavy forging defects in the upsetting process focus on both the surface crack ${ }^{[16-18]}$ and closing/bonding of inner void ${ }^{[19-24]}$ of the forgings in recently years. Based on the above, there is not a uniform interpretation to the inner laminated crack of large forgings and its criterion model yet. So it is of great significance on the detailed theoretical analysis of its evolution of deformation mechanics.

With the increasingly maturity of finite element analysis (FEA) technology, it has become the consensus that finite element method (FEM) is selected as a powerful tool to solve many practical problems. In the past years, commercial FEA software MARC was used to simulate the closing process of the inner crack in cylindrical body during hot upsetting, analyse the stress and strain of the crack during deformation, and various factors that affect closing and bonding of the inner crack ${ }^{[25]}$. Banaszek ${ }^{[19,20]}$ studied the upsetting process of a cylinder with a void by the commercial FEA software Forge and through simulation investigate the influence of forging processing on the internal void closure of the forgings and gain the reasonable forging conditions of defect-free. Based on DEFORM software, the coupling thermal-mechanical model of upsetting process was established separately by Zhang ${ }^{[17]}$ and Huang ${ }^{[26]}$, and to investigate the effect of upsetting parameters on centre compaction and void changing. According to simulated results, improving measures were proposed. A coupling thermo-mechanical model of heavy disk-shaped forgings in the upsetting process of was established using rigid-plastic finite element method in this study. Based on the model, the evolution of stress-strain state and the deformation gradient distribution in the upsetting process was presented as for the ratio of height to diameter greater than $2(H / D>2)$, and then the formation mechanism of laminated crack defect was investigated. At last, a criteria model for laminated crack of the disk-shaped forgings was proposed and verified.

\section{Finite element model}

\subsection{The basic theories of the rigid-viscoplastic thermal-mechanical finite element model}

The analysis of the upsetting process makes use of the rigid-plastic FEM based on the flow formulation of the penalized form of the incompressibility. According to the variational principle, the basic equation for the finite-element formulation is expressed as:

$$
\int_{V} \bar{\sigma} \delta \dot{\bar{\varepsilon}} d V+K \int_{V} \dot{\varepsilon}_{v} \delta \dot{\varepsilon}_{v} d V-\int_{S_{F}} F_{i} \delta u_{i} d S=0
$$

where $\bar{\sigma}$ is the equivalent stress, $\dot{\bar{\varepsilon}}$ is the equivalent strain rate, $F_{i}$ represents surface tractions, $u_{i}$ the velocity field, $K$, a penalty constant, is a very large positive constant, $\dot{\varepsilon}_{v}=\dot{\varepsilon}_{i i}$, is the volumetric stain rate.

For studying the plastic deformation behavior of a given metal it is appropriate to consider uniform or homogeneous deformation conditions. The yield stress of a metal under uniaxial conditions as a function of strain, strain rate and temperature can also be considered as flow stress. The metal starts flowing or deforming plastically when the applied stress reaches the value of yield stress or flow stress. It is assumed that the material is isotropic and that the yielding behavior follows the Levy-Mises yield criterion. The Levy-Mises flow rule can be expressed as:

$$
\sigma_{\mathrm{ij}}=\frac{1}{\dot{\lambda}} \dot{\varepsilon}_{\mathrm{ij}}
$$

where $\sigma_{i j}$ and $\dot{\varepsilon}_{i j}$ are the component of stress tensor and the component of strain rate tensor, respectively. 


$$
\dot{\lambda}=\frac{3 \dot{\varepsilon}_{e}}{2 \sigma_{e}}
$$

Where $\dot{\varepsilon}_{e}$ and $\sigma_{e}$ are the effective strain rate and effective stress, respectively.

The analysis of heat transfer can also be readily carried out by solving the following energy balance equation.

$$
k \nabla^{2} T+\dot{q}-\rho c \dot{T}=0
$$

where $k, \rho, c, \dot{q}$ and $T$ denote the thermal conductivity, specific density, specific heat, heat generation rate and temperature, respectively. The first term $k \nabla^{2} T$ and the third term $\rho c \dot{T}$ represent the heat-transfer rate and the heat-generation rate, respectively. The rate of the heat generation in the deformed body due to plastic deformation is given as:

$$
\dot{q}=\alpha \bar{\sigma} \dot{\bar{\varepsilon}}
$$

where the heat-generation efficiency, $\alpha$, represents the fraction of mechanical energy converted into heat, and is usually assumed to be 0.9 . The energy equation, Eq. (4), can be rewritten using the weighted residual method as:

$$
\int_{V} k T_{, i} \delta T_{, i} d V+\int_{V} \rho c \dot{T} \delta T d V-\int_{V} \alpha \bar{\sigma} \dot{\bar{\varepsilon}} \delta T d V-\int_{S} q_{n} \delta T d s=0
$$

where $q_{\mathrm{n}}$ is the heat flux normal to the boundary surface. The temperature distribution of the forgings can be obtained readily by solving the above energy balance equation.

Two friction types, coulomb model and shear model, are often used. Constant shear friction model is used mostly for bulk-forming simulations. The frictional force in the constant shear model is defined by:

$$
f_{S}=m k
$$

where $f_{s}$ is the frictional stress, $k$ is the shear yield stress and $m$ is the friction factor. This states that the friction is a function of the yield stress of the deforming body.

\subsection{The finite element model for upsetting with laminated defect}

$\mathrm{Cr} 5 \mathrm{MoV}$ material is selected as a research object in this work, combining with actual technology parameters, and stress-strain curves are obtained via thermal-mechanical simulation experiment, as shown in Fig. 1. Based on the deformation characteristics of cylinder upsetting between flats, a quarter axisymmetric coupling thermal-mechanical model is built using non-linear FEM, as shown in Fig. 2, where the size $H \times D$ of large forged billet is $2840 \mathrm{~mm} \times \Phi 1220 \mathrm{~mm}$. Laminated defect will be presented using birth-death element in this model. Calculation parameters are listed in Table 1.

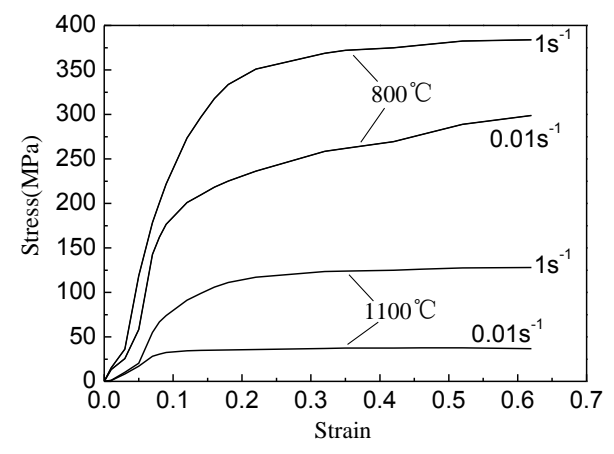

Fig. 1 True stress-strain curve

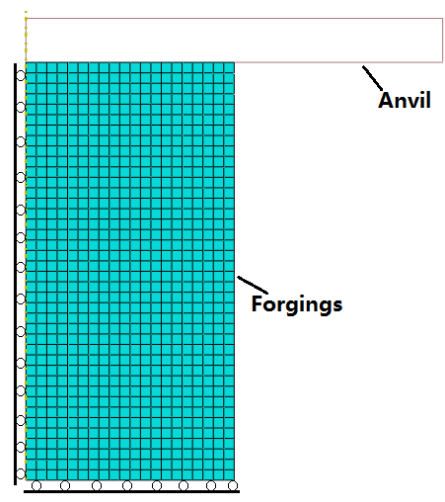

Fig.2 Finite element model

Table 1 Processing and simulation parameters

\begin{tabular}{l|l}
\hline parameters & value \\
\hline
\end{tabular}




\begin{tabular}{l|l}
\hline Initial temperature of forging $\left(\left({ }^{\circ} \mathrm{C}\right)\right.$ & 1100 \\
\hline Initial temperature of anvil $\left(\left({ }^{\circ} \mathrm{C}\right)\right.$ & 200 \\
\hline Speed of anvil $(\mathrm{mm} / \mathrm{s})$ & 20 \\
\hline Friction coefficient & 0.7 \\
\hline Contact heat transfer coefficient $\left(\mathrm{N} / \mathrm{s} / \mathrm{mm}^{2}\right)$ & 20 \\
\hline Convective heat transfer coefficient $\left(\mathrm{N} / \mathrm{s} / \mathrm{mm}^{2}\right)$ & 0.02 \\
\hline Black-body radiation coefficient & 0.8 \\
\hline Environment temperature $\left(\left({ }^{\circ} \mathrm{C}\right)\right.$ & 30 \\
\hline
\end{tabular}

\section{Introduction to tensile stress theory of rigid-plastic mechanics}

In order to determine the internal deformation mechanism and variation of stress state during upsetting, Liu ${ }^{\text {[7] }}$ divided forgings into several different areas to study and proposed a tensile stress theory of rigid-plastic mechanics. The model of rigid-plastic mechanics is shown in Fig. 3 with H/D>2 during forging upsetting, where I is cone-shaped rigid zone, II is active plastic deformation zone, III is passive plastic deformation zone, and IV is uniform compression zone. In Fig. $3, \sigma_{\mathrm{z}}$ is axial stress, $\sigma_{\mathrm{r}}$ radial stress, and $\sigma_{\theta}$ circumferential stress. Stress state in II zone is two-direction compressive stress $\left(\sigma_{\mathrm{r}}\right.$ and $\left.\sigma_{\mathrm{z}}\right)$, one-direction tensile stress $\left(\sigma_{\theta}\right)$. This stress state can meet Tresca Yield Criterion Formula (8).

$$
\sigma_{1}-\sigma_{3}=\sigma_{\theta}+\left|\sigma_{\mathrm{z}}\right|=\sigma_{\mathrm{s}}
$$

Stress state in III zone is two-direction tensile stress $\left(\sigma_{\mathrm{r}}\right.$ and $\left.\sigma_{\theta}\right)$, one-direction compressive stress $\left(\sigma_{\mathrm{z}}\right)$, and there is no starting trend of plastic deformation.

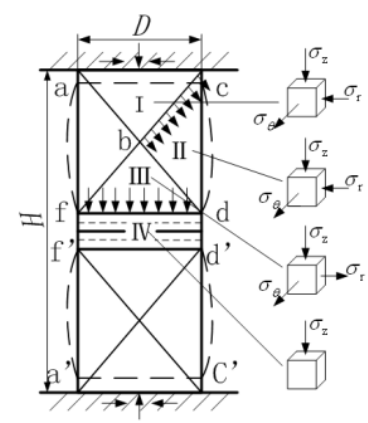

Fig. 3 Model of rigid-plastic mechanics with H/D $>2$ during upsetting

The theory assumes that the shape and range of rigid zone do not change in the upsetting process, and Tresca Criterion is used as material yield plastic formula. To more realistically predict the deformation and stress state of forging, a coupling thermal-mechanical finite element model for whole upsetting process was established and analyzed in this study.

\section{Analysis on regional differences of deformation mechanism}

Based on the calculated results from the above model, the equivalent strain distribution (Fig.4) indicates that deformation of the forgings is very inhomogeneous throughout the upsetting process, and deformation degree has a strongly regional difference. As a result of the effect of external friction from the contact area with the anvil, limiting the metal flow, two upper and lower symmetrical larger cone-shaped rigid zones are formed. The range of cone-shaped rigid can be reduced by decreasing the friction. According to the rigid-plastic theory, the metal in the drum area below the corners (zone II near the edge) easily deforms during upsetting, but equivalent strain in this area is always less than that in the centre. The equivalent strain rate distribution in Fig.5 shows that 
the equivalent strain rate in this region increases slowly than that in the centre.

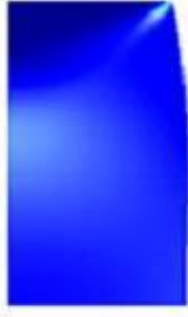

(a) Reduction of $20 \%$

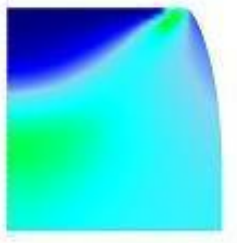

(b) Reduction of $40 \%$

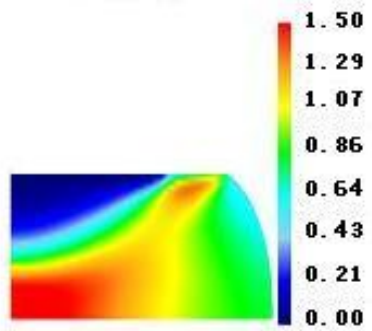

(c) Reduction of $60 \%$

Fig. 4 Cloud diagram of equivalent strain varies with reduction

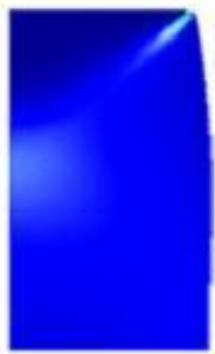

(a) Reduction of $20 \%$

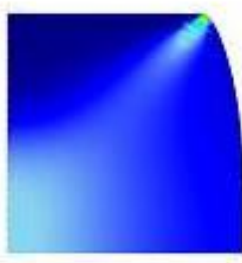

(b) Reduction of $40 \%$

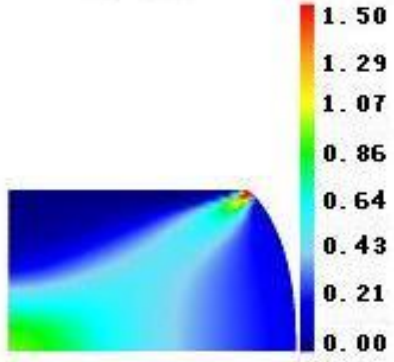

(c) Reduction of $60 \%$

Fig. 5 Cloud diagram of equivalent strain rate varies with reduction

Observation on the change of equivalent strain in the axis (Fig. 6) shows deformation in the end of the deformable body is weak and deformation in the rigid area increases slowly with the development of upsetting. Therefore, the range of rigid area does not obviously decrease with the increase of reduction. However, equivalent strain below the rigid area sharply increases, and deformation closer to the centre is more significant. At the beginning stage of upsetting, as a result of the effect of rigid zone, the equivalent strain in front of the rigid zone reaches the peak and then reduces when it is near the centre. This means that the maximum value of equivalent strain in the axis is not exactly at the centre, and the maximum value shifts towards the centre with the increase of reduction. The maximum equivalent strain is gained in the centre when reduction increases to a certain extent. Especially, after reduction reaches 50\%, equivalent strain in the centre rapidly rises, which indicates that the deformation rate increases at this point.

As for long cylinder forgings of height-diameter ratio $H / D>2$, besides the corner point, the maximum of equivalent strain occurs below the cone-shaped rigid zone, and moves towards the centre with the development of upsetting. So, the position firstly enters into plastic state in the upsetting process.

The gradient of equivalent strain $\nabla \bar{\varepsilon}$ in the axial direction was calculated and shown in Fig.7. According to the distribution results, the gradient of equivalent strain is almost zero at the top of forging, and then increases gradually, and later it increases suddenly until the distance from the top of forging reaches a certain value. Subsequently, it quickly falls near zero after the peak value of $\nabla \bar{\varepsilon}$. So, a peak point of equivalent strain gradient occurs. Moreover, the change of the strain rate is increasingly obvious as the reduction increases. The peak rapidly increases and moves to the centre after the reduction is more than $60 \%$. The greater equivalent strain gradient is at the peak, the more significant metal deformation at that position.

Fig. 8 shows the gradient distribution of equivalent strain rate $\nabla \dot{\bar{\varepsilon}}$ under the corresponding condition, of which the change trend is basically similar to that of gradient of equivalent strain and both gradient peaks occurred roughly at the same location. Because of the gradient changing of equivalent strain rate, the metal deformation 
rate varies at the different positions, and the gradient variation is more significant especially near the peak area as shown in Fig. 8, which leads to inhomogeneity of order and speed of metal flow, poor coordination of material deformation, as a result, it is easy to cause material damage.

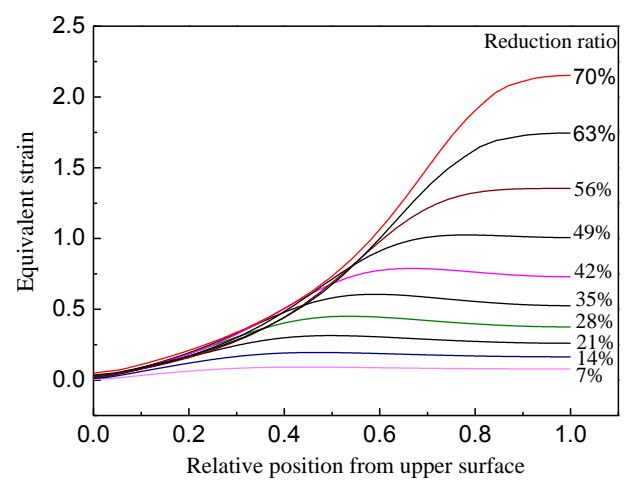

Fig. 6 History curve of equivalent strain in the axis

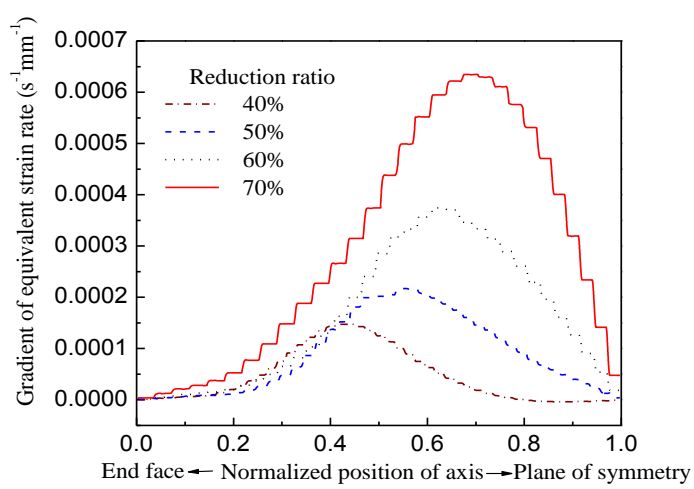

Fig. 8 Gradient of equivalent strain rate in the axial

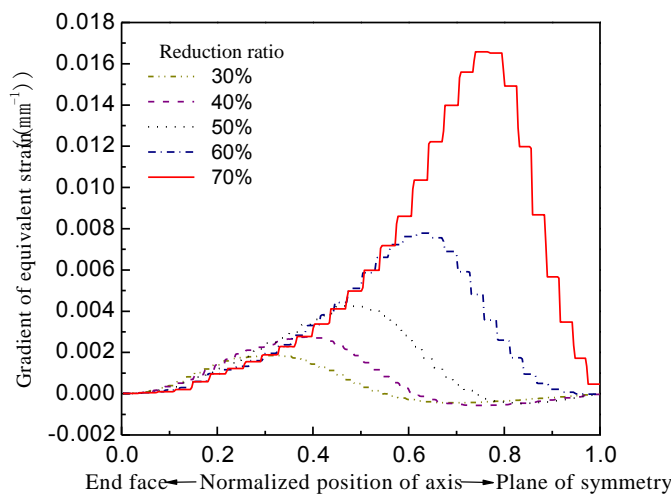

Fig.7 Gradient of equivalent strain in the axial

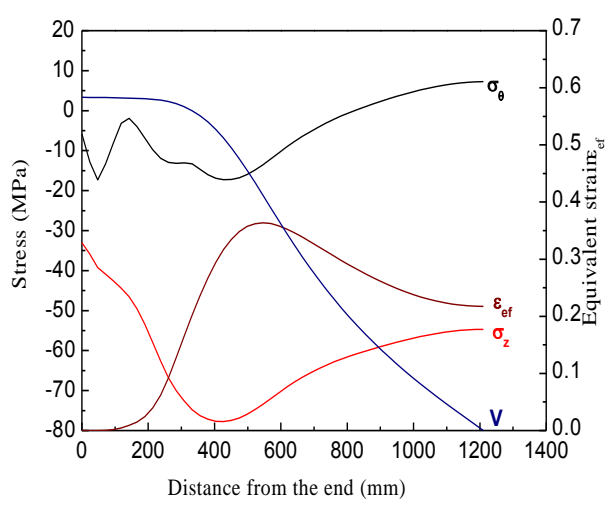

Fig. 9 Distributions of field variables in central axis with reduction of $20 \%$

Fig. 9 shows the distributions of different field variables on the central axis of forging as the reduction is $20 \%$. The results indicate that the position of maximum absolute value of stress in the direction of forging $\left(\sigma_{z}\right)$ is basically the same with that of maximum slope of equivalent strain rate curve.

Fig. 10 shows the distributions of the axial stress $\left(\sigma_{z}\right)$ and the circumferential stress $\left(\sigma_{\theta}\right)$ in the cross section of maximum equivalent strain with different reductions. Combination of Figs. 9 and 10, it can be seen that either on a central axis or on the diameter location corresponding to the largest equivalent strain, maximum absolute values of stress $\left(\sigma_{z}\right)$ in the direction of forging are all located near the position of the maximum equivalent strain, which is the intersection area of I, II, III zones in Fig. 3, and in tri-lateral compressive stress state. By comparison of the stress $\sigma_{\mathrm{z}}$ with the stress $\sigma_{\theta}$, the absolute value of $\sigma_{\mathrm{z}}$ is much larger than $\sigma_{\theta}$. Therefore, the stress $\sigma_{z}$ has a significant influence on the establishment of Equation (8), while the stress state here has little influence on Equation (8). Compared with rigid-plastic tensile stress theory, the simulated results from finite element model indicate the intersection area of I, II, III zones first enters plastic state and reaches the maximum deformation, where the stress state is tri-lateral compression. 


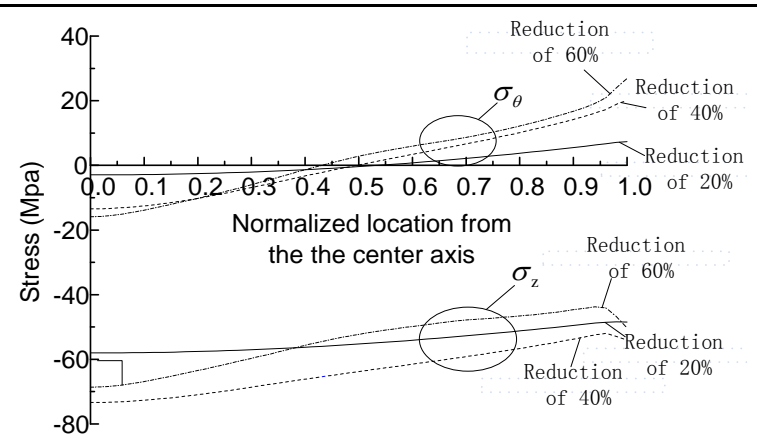

Fig. 10 distribution of stress along the radial direction in the cross section of maximum equivalent strain

\section{Evolution law of stress state}

In the upsetting process, because the stress $\sigma_{\mathrm{z}}$ in the axis direction is always negative, which means compressive stress, stresses of other two directions, to a large extent, determine the stress state here, and the complex change of circumferential and radial stresses are also caused as the ratio of height to diameter changes. Based on simulated and analyzed results from coupling thermo-mechanical finite element model for upsetting the evolution process of the stress state insides the forgings was obtained and shown in Fig. 11 by aid of a visual method describing the internal stress state regionally.

Fig. 11 shows the distribution of internal stresses when relative reductions are 0.2, 0.4, and 0.6 respectively. In region A of Fig.11, radial stress $\left(\sigma_{\mathrm{r}}\right)$ and circumferential stress $\left(\sigma_{\theta}\right)$, the axial stress $\left(\sigma_{\mathrm{z}}\right)$ are all negative, namely tri-lateral compressive stresses. $\sigma_{\mathrm{r}}$ and $\sigma_{\mathrm{z}}$ are negative, and $\sigma_{\theta}$ positive in region B, which are two compressive stresses and one tension stress. In region $\mathrm{C}, \sigma_{\mathrm{r}}$ and $\sigma_{\theta}$ are positive, and $\sigma_{\mathrm{z}}$ is negative, which are two tensions and one compression. In region $\mathrm{D}, \sigma_{\mathrm{r}}$ is positive, $\sigma_{\theta}$ and $\sigma_{\mathrm{z}}$ negative, which are two compressive stresses and one tension. It can be seen obviously in Fig. 11 that with the increasing of reduction, the range of central region $\mathrm{C}$ shrinks to the centre; regions $\mathrm{A}$ and $\mathrm{B}$ extend towards the centre correspondingly. When relative reduction reaches around $40 \%, H / D \approx 1.0$, region $C$ near the centre will be turned into regions $A$ and $\mathrm{B}$, as a result radial and circumferential stresses are changed from tensile stress to compressive stress. At this time, the centre is in the state of three-dimensional compressive stresses. Ultimately, only regions A and B are left, which is compressive stress in central region, and at the circumferential region, the circumferential stress is tensile, and other stresses are compressive in the radial and forging directions.

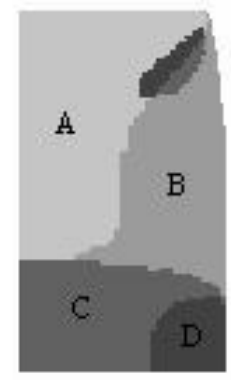

(a) Reduction ratio of $20 \%$

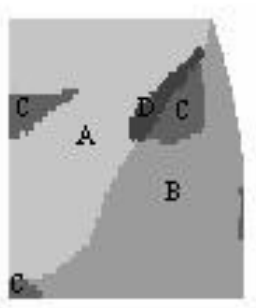

(b) Reduction ratio of $40 \%$

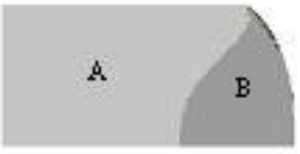

(c) Reduction ratio of $60 \%$

$$
\left(A: \sigma_{\mathrm{r}}-、 \sigma_{\theta^{-}}, \sigma_{\mathrm{z}}-; \quad B: \sigma_{\mathrm{r}}-, \sigma_{\theta}+, \sigma_{\mathrm{z}}-; \quad C: \sigma_{\mathrm{r}}+, \sigma_{\theta}+, \sigma_{\mathrm{z}}-; \quad D: \sigma_{\mathrm{r}}+, \sigma_{\theta^{-}}, \sigma_{\mathrm{z}}-\right)
$$

Fig. 11 Evolution of stress distributions 
That stress state in the centre of the forging is gradually changed from two compressive stresses and one tension into three-dimensional compressive stresses. So it indicates stress state is beneficial to compaction of centre, however it cannot explain forming cause of the inner laminated crack.

\section{Laminated crack defect}

\subsection{Criterion of ductile fracture}

Aiming at failure mode of engineering materials and structures, many kinds of strength theories have been proposed. Besides, many scholars have proposed different forms of expression on criterion of ductile fracture about local material failure of workpiece in metal plastic forming process. Different damage results were obtained and shown in Table 2 after the finite element modelling of cylinder upsetting between flats were carried out using the above criterions. Table 2 shows the damage distributions in symmetry plane using different criterions of material ductile fracture in the upset forging process. Through the comparison of the above results, it can be found, except Freudenthal Criteria, other criteria's damage results are basically larger within circumferential drum area. The maximum principal stress is regarded as dominant mechanics factor of material failure for most of these criteria, which can describe crack on the surface of drum-type area of the upsetting cylinder, however cannot explain the laminated crack defect within the forgings. Freudenthal Criteria reflects the plastic work of material deformation. When the effect of friction is ignored during upsetting, cylindrical workpiece is the single compression deformation, and the laminated crack defect will not occur, but the plastic work evenly distributes in the workpiece, and large value can be achieved too. To summarize, this criterion cannot be used for the defect.

\subsection{Establishment of laminated crack model}

By the above qualitative analysis, deformation in the front of the rigid area has a significant effect on the production of laminated crack defect. Finite element simulation of the forging process is used for further research on the deformation mechanism of the front of rigid area under large reduction. Based on the analysis of equivalent strain of forgings during upsetting, the contour line distribution of the equivalent strain in Fig. 12 indicates that with the increase of reduction, contour line around cone-shaped top of the rigid area becomes dense, this means that the gradient of equivalent strain increases. Especially after reduction reaches $60 \%$ and $70 \%$, contour line in front of the rigid area increases and becomes very dense. Throughout the upsetting process, contour line in front of the rigid area is continuously in the densest state, so it can be believed that the metal flow is more intense here. In addition, as the front of rigid area gradually turns into plastic state, cone shape of rigid area becomes flat. Variation of distribution of equivalent strain on the central axis of the forgings shown in Fig. 12 indicates that from the top to the centre of the forging, the strain increased gradually from remarkably and this non-uniform variation will cause inconsistence of deformation in the adjacent metallic layers.

Table 2 Damage results of different criterion

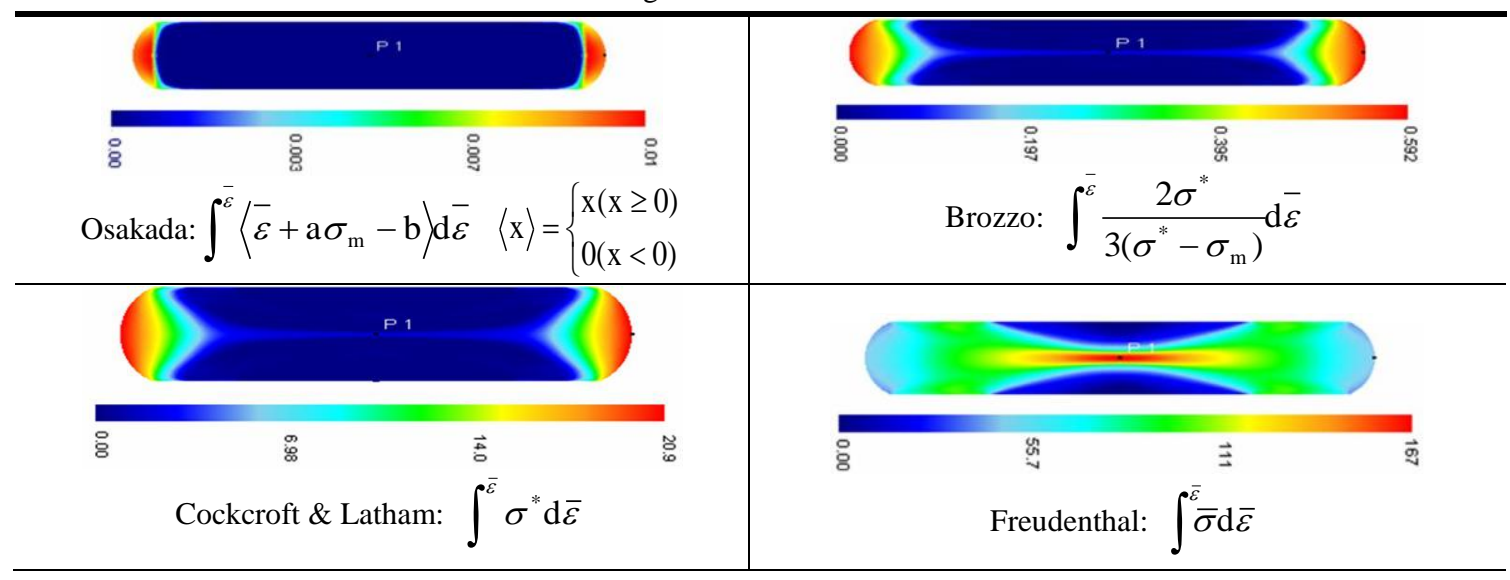




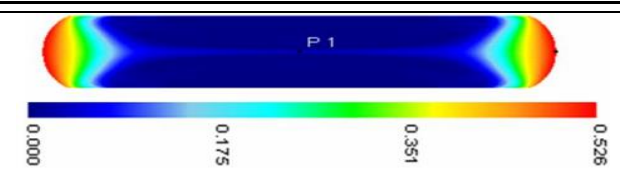

Normalized Cockcroft \& Latham: $\int^{\bar{\varepsilon}} \frac{\sigma^{*}}{\bar{\sigma}} \mathrm{d} \bar{\varepsilon}$

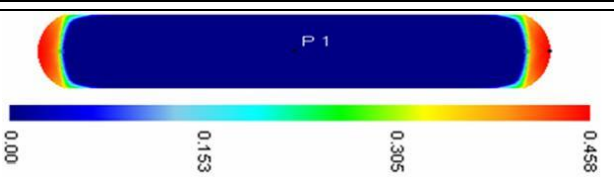

Rice \& Tracy: $\int^{\bar{\varepsilon}} \mathrm{e}^{\frac{\alpha \sigma}{\bar{\sigma}} \mathrm{d}} \bar{\varepsilon}$

(Where $\sigma^{*}$ is the maximum principal stress, $\bar{\sigma}$ is equivalent stress, $\sigma_{\mathrm{m}}$ is average stress, ${ }{ }_{\text {is equivalent strain) }}$

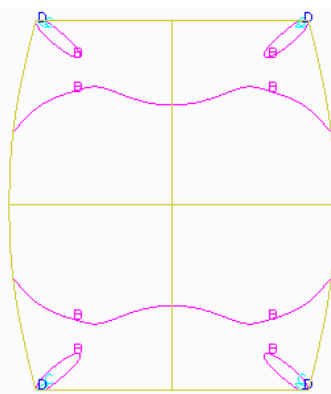

(a)Reduction ratio of $30 \%$

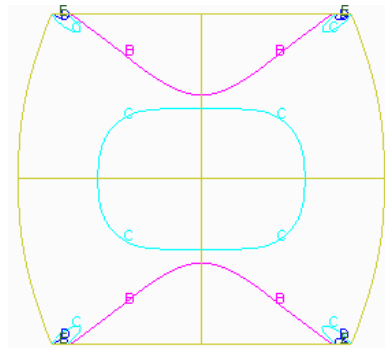

(b)Reduction ratio of $40 \%$

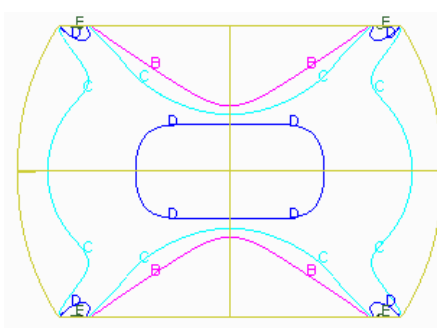

(c)Reduction ratio of $50 \%$

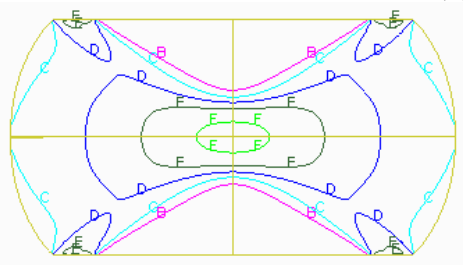

(d)Reduction ratio of $60 \%$

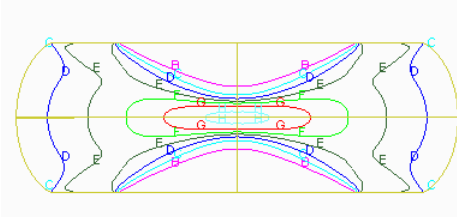

(e)Reduction ratio of $70 \%$

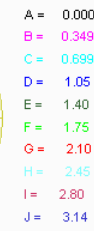

$J=3.14$

Fig. 12 Contour line chart of the equivalent strain

The equivalent strain rate on the central axis $(\dot{\bar{\varepsilon}})$, as shown in Fig.13, is similar to the distribution of equivalent strain, namely the change of $\dot{\bar{\varepsilon}}$ from the top to the horizontal symmetry is first slowly, then faster, and then slower increases again. After reaching a certain value, it remains basically unchanged. In the range away from the horizontal symmetry plane, equivalent strain rate remains relatively high, taking reduction of $40 \%$ for instance, there is basically a large unchanging range of equivalent strain rate, and as the reduction increases, the value of $\dot{\bar{\varepsilon}}$ increases, however, the scope of the unchanging reduces. It indicates deformation rapidly increases within a very small region close to the horizontal symmetry plane when a larger reduction is applied. So, it is consistent with the analysis results of equivalent strain.

According to the curves in Fig. 13, it is evaluated to obtain the gradient changing of equivalent strain rate ( $\nabla \dot{\bar{\varepsilon}})$ in the axis direction, as shown in Fig. 8. A peak of the gradient $\nabla \dot{\bar{\varepsilon}}$ change always occurs under different reductions and moving towards the centre as the reduction increases, and the peak value increases as the reduction increases, and the changing amplitude of the gradient increases obviously after the reduction reaches $60 \%$ especially. Due to the different gradients of equivalent strain rate, speeds of metal deformation at different locations are different, and the difference is more serious in various locations close to peak area shown in Fig. 8, and the difference would lead to non-uniform of order and speed of metal flow, less coordination of material deformation, and easily cause material damage. 


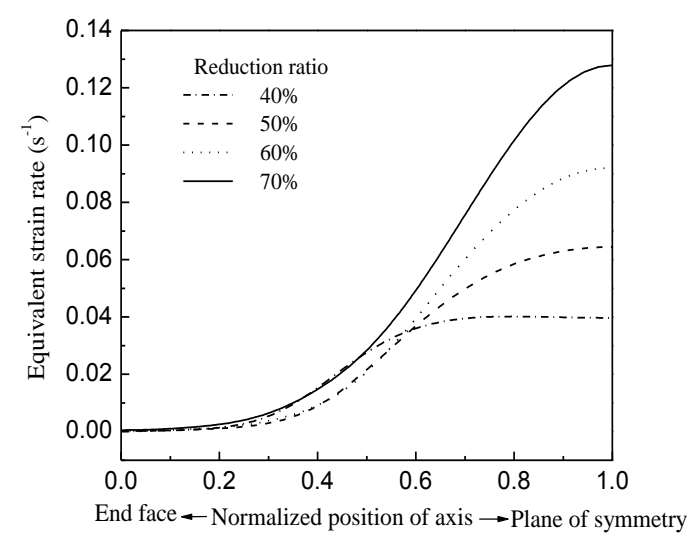

Fig. 13 Distribution in axial direction of equivalent strain rate

\subsection{Model of laminated crack defect}

The above analysis indicates when upsetting under reduction over $60 \%$, the equivalent strain $(\bar{\varepsilon})$ and its gradient $(\nabla \bar{\varepsilon})$, equivalent strain rate $(\dot{\bar{\varepsilon}})$ and its gradient $(\nabla \dot{\bar{\varepsilon}})$ on a central axis have taken place major changes, especially values of $\nabla \bar{\varepsilon}$ and $\nabla \dot{\bar{\varepsilon}}$ reach large peak values, and narrow range of peaks. Odd points of these field variables occur in the basic consistent location: the peak location of $\nabla \bar{\varepsilon}$ and $\nabla \dot{\bar{\varepsilon}}$, where, after reduction of $60 \%$, larger metal deformation occurs, according to value of field variables and variation, and degree and speed of deformation are greatly not coordinated, which occurs in a narrow range. Under tri-axial stress state, lack of coordination between layers of metal would easily lead to relative slip and slip tear appears, which causes the inner laminated crack defect in the forgings. It is similar to the phenomenon of laminated crack defect often appears in large tube-sheet workpiece. For such a unique phenomenon and upsetting technology for large tube-sheet workpiece, based on the above analysis of un-coordination of material deformation in space and time, active effect of gradient of degree and speed of deformation on damage to materials, combination of relations in Equation (9) represents the model of forming of laminated crack defect.

$$
\left\{\begin{array}{c}
\bar{\varepsilon} \geq \mathrm{C}_{1} \\
\nabla \dot{\bar{\varepsilon}} \geq \mathrm{C}_{2}
\end{array}\right.
$$

where $\mathrm{C}_{1}$ and $\mathrm{C}_{2}$ are respectively the critical value of gradient of equivalent strain and equivalent strain rate when laminated defects occurs, they are selected at the peak of gradient of equivalent strain rate, and their value is related to characteristics and deformation temperature of upsetting material. Based on the above analysis of field variables and actual production conditions, reduction of about $65 \%$ is selected here $(\mathrm{C} 1=2.5$ and $\mathrm{C} 2=0.0005$ ). The above relation model is embedded into finite element model to simulate forming of upsetting laminated crack. Fig.14 shows the situation of internal damage when reduction reaches $65 \%$. It can be obviously seen that on both sides of the central symmetric plane, two damaged bands occur, as well as crack. Therefore, the above model of dissection cracks appearance simulation, can intuitively reconstruct morphology and distribution of laminated crack in the centre of forging part.

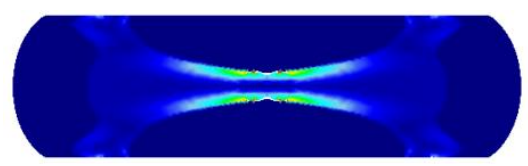

Fig. 14 Damaged image of laminated crack defect

The model is based on actual working condition, established on study of deformation mechanism in the regional where laminated crack defect easy occurs, and obtained by integrated analysis of variation of all deformation 
field variables, therefore, it is only limited to technology range of upsetting of large tube-sheet workpiece. However, it can be used as the index variable in the numerical simulation process of upsetting to intuitively figure out location and degree that laminated crack defect occurs, and provides reference for actual production line.

\subsection{Experimental verification}

In order to verify that reasonableness and accuracy of the combination model, casting aluminum rod with size of $\Phi 38 \mathrm{x} \times 80 \mathrm{~mm}$ is used in upsetting test in this study. Workpiece is preheated to $300^{\circ} \mathrm{C}$, different reductions, respectively 50\% and 65\%, were carried out in the upsetting and the results are shown in Fig. 15.

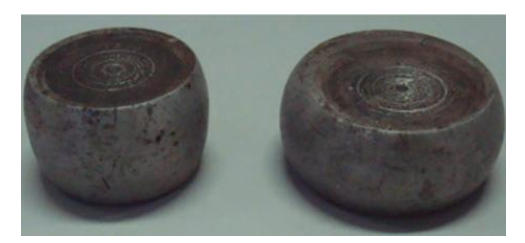

Fig. 15 Upsetting results under different reductions

According to simulation results of finite element modelling based on experimental conditions, critical parameters in Equation (9) are selected as corresponding values locating at the peak of equivalent strain rate gradient on the axis. After modelling with reduction of $65 \%$ was carried out, the simulation result of crack morphology distribution was obtained and shown in Fig. 16.

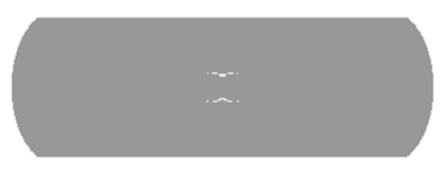

Fig. 16 Simulation result of morphology and distribution of laminated crack

The upsetting test workpiece was anatomized along the central axis, and observed along the central axis under the optical microscope after polishing the section. The workpiece with reduction of $50 \%$ is not unusual, however, crack was found in workpiece with reduction of 65\%, as shown in Fig.17. Black spots in the base material shown in Fig. 16 are the forged porosity defects. At the position of severe deformation in the axial direction, including drastic changes in the spatial location and deformation rate, pores are torn to expand and form crack, even for homogeneous materials without flaws, this location is also prone to be damaged under the same conditions. Fig.17 shows the location and morphology of cracks, coinciding well with the simulation result in Fig. 16, which indicates that the determining method of expression and threshold of model in Equation (9) can be used to predict the laminated crack defects in upsetting process.

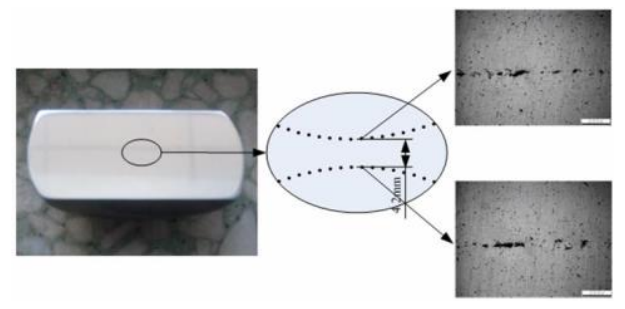

Fig. 17 Experimental result of morphology and distribution of laminated crack

\subsection{Effect of friction coefficient on the deformation}

Friction coefficients between forging and anvil are selected as $0.3,0.5$, and 0.7 , respectively. The result of finite 
element simulation was analyzed and shown as equivalent strain distribution in Fig. 18, which obviously shows that the rigid areas occur in both ends of forging. As for the same reduction, rigid zone in the radial and axial directions are both increasing with the increase of friction coefficient, but that increases significantly in the axial direction and modestly in the radial direction. As rigid areas increases, meanwhile range of internal high-strain zone also increases. Effects of friction status between the anvil and workpiece on deformation state in the workpiece are complex. According to Fig. 19, effects of different friction coefficient on equivalent strain on the axis of workpiece can be divided into two parts. The results show that the equivalent strain is large when the friction coefficient is small in the first half of near the end, and contrary to the second half close to horizontal symmetry plane.

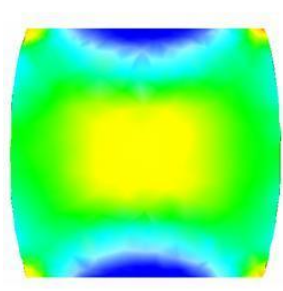

(a) $\mathrm{f}=0.3$

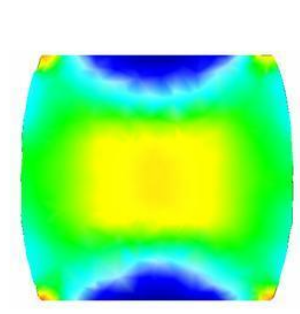

(b) $\mathrm{f}=0.5$

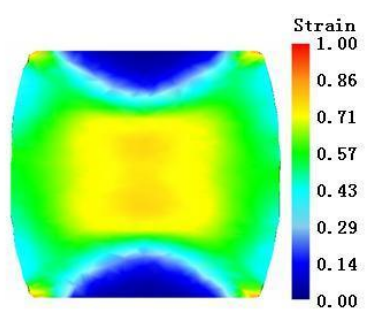

(c) $\mathrm{f}=0.7$

Fig. 18 Effective strain contour by different friction coefficient

Curve of equivalent strain distribution on the central axis (Fig. 19) also shows large friction coefficient leads to the equivalent strain decrease in the rigid area, but equivalent strain rapidly increases away from the rigid area, which leads to a large internal equivalent strain. For example when the friction coefficient is 0.7 , the increase of equivalent strain at both ends is extremely slow towards the centre resulting in a large range of rigid area. However, equivalent strain grows quickly. It can be been seen that when the friction coefficient is large, the end is uneasy to deform but beneficial to deformation in the centre, especially when the reduction increases, deformation in the centre increases significantly, which helps to improve the quality of forging components.

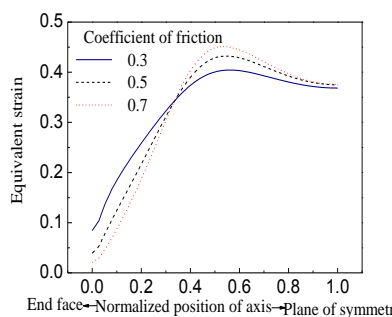

(a) Reduction of $28 \%$

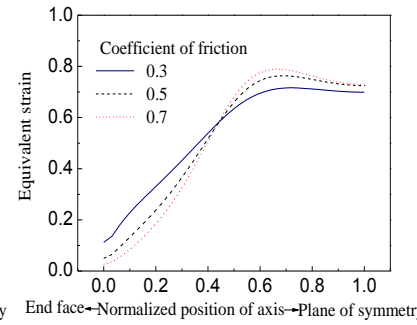

(b) Reduction of $42 \%$

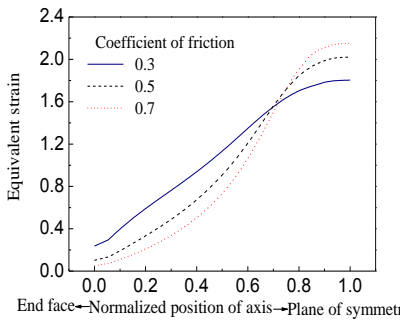

(c) Reduction of $56 \%$

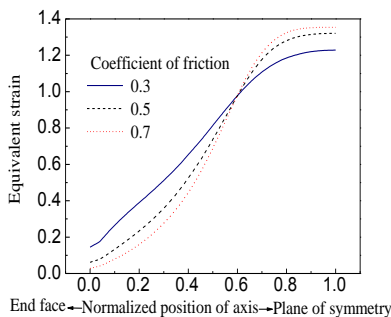

(d) Reduction of $70 \%$

Fig. 19 Effect of friction coefficient on the effective strain

As rigid zone is defined as the zone where equivalent strain is less than 0.1 and distance from the vertex of cone-shaped rigid area to the end is taken as the depth of rigid zone, the curve of friction coefficient variation is drawn in Fig. 20. It can be found from Fig. 20(a) that as friction coefficient increases with the same reduction, depth of rigid zone increases. As for small reduction, depth of rigid zone uniformly increases, but in a small amplitude as the friction coefficient increases; when the reduction increases, depth of rigid zone in small friction coefficient decreases rapidly, additionally as friction coefficient increases, the increased amplitude of rigid area depth is first large and then becomes small. Curve in Fig. 20(b) shows relation between the depth of rigid zone and percentage of current distance from centre to the top of the forging with different friction coefficients, which can be used to indicate the range of rigid zone in the current forging in upsetting process. It can also be 
seen from Fig. 20 when the friction coefficient is larger, the increase of reduction has little effect on current range of rigid zone in forging; while the friction coefficient is smaller, and the rigid area significantly decreases as the reduction increases. Therefore, rigid area changing with low friction coefficient is sensitive as to reduction of upsetting and proportion of rigid area is basically the same under high friction coefficient, only after reduction grows to $50 \%$, range of rigid area decreases significantly.

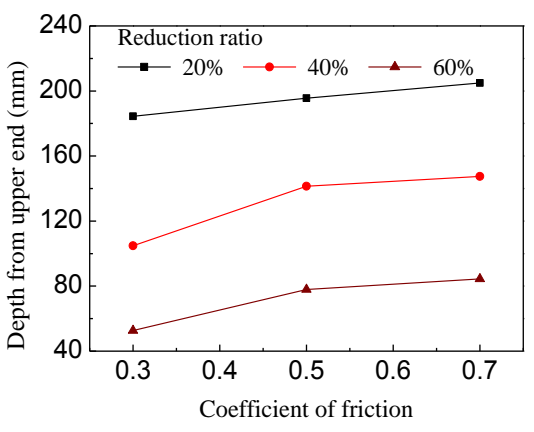

(a) Absolute depth of rigid zone

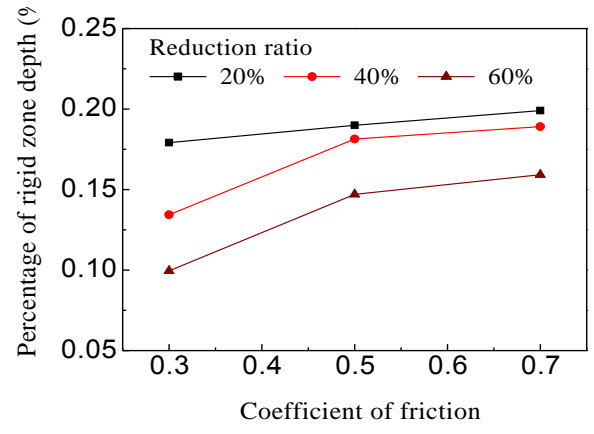

(b) Percentage of rigid zone depth

Fig. 20 Rigid zone depth by different friction coefficients

According to the above analysis, it can been seen that the increase of friction coefficient leads to increase of rigid area, but when the friction coefficient increases to a certain value, the range of rigid zone increases in a small amount, this means that the effect of friction coefficient on the range of rigid zone is weak, but the deformation degree increases in the centre of workpiece. Although increasing friction coefficient can increase the deformation degree of the centre of workpiece, as for the large friction coefficient the decrease of rigid area must be augmented by large reduction. Besides, it will make deformation gradient below the rigid area change more dramatically, which will cause laminated crack defect easily.

\section{Conclusions}

(1) In the upsetting process of large cylindrical body between flats, the absolute value of axial stress is large, which is the main factor that effects the establishment of Tresca yield criterion. Maximum strain occurs in centre axis, and in the region locating at the front of cone-shaped rigid area, where is Triaxial Compressive Stress State.

(2) In the upsetting process of large forging, metal flow speed of where the maximum equivalent strain occurs sharply reduces, metal accumulates here, and axial stress increases. So, plastic deformation easily occurs first near junction of I, II and III areas.

(3) Under the ratio of height to diameter (H/D)>1, $\sigma_{\mathrm{r}}$ and $\sigma_{\theta}$ are tensile stresses, and $\sigma_{\mathrm{z}}$ is compressive stress in the central area, and this area contracts towards the centre during upsetting; this area disappears around the $\mathrm{H} / \mathrm{D}=1$, and ultimately stress state presents three-directional compression in the centre, while the edge circumferential stress is tensile, and stresses pressure on radial and forging direction in the edge area.

(4) It was proposed that the difference of deformation gradient in the upsetting process of large forgings will lead to inhomogeneity of order and speed of metal flow, and poor compatibility of material deformation, which is the main reason of laminated crack defect.

(5) According to gradient of equivalent strain and gradient of strain rate, a combined estimation model of laminated crack defect was established and successfully obtains morphology and distribution of laminated crack in the centre of forgings. The reliability criterion of the model is verified by aid of physical simulation experiment of small forgings.

(6) Increasing friction coefficient causes laminated crack defect easily.

\section{Acknowledgments}


This research has been supported by the National Natural Science Foundation Project of China (No.51075352), China Postdoctoral Science Foundation (No.135877), Iron and Steel Union Program of Natural Science Foundation of Hebei Province(No.E2015203343) and Independent Research Project of Yanshan University (No.13LGA002). The sponsorships are gratefully acknowledged.

\section{References}

[1] Yulin Shi. Study on Forging Process of the Squeeze Roll of Whole Forging . Wuhan. Huazhong University of Science and Technology, 2006.

[2] Danian Wang. Principles of Metal Forming. Beijing: China Machine Press,1986:217-236.

[3] Hongde Cao. Plastic deformation mechanics basis and principle of rolling. Beijing: China Machine Press, 1983:113-118

[4] Zhubai Liu, Liandong Wang, Guohui Liu. Physical Simulation of Tension Stress Theory of a Rigid Plastic Mechanical Model for Upsetting a Circular Cylinder. Chinese Journal of Mechanical Engineering, 1993, 6(2):102-105.

[5] Zhubai Liu, Liyong Ni, Chen Liang, Guohui Liu, Dongmei Deng. Simulation of Tensile Stress Theory of Conventional Upsetting between Flat Plates for High Cylindrical Forgings. China Mechanical Engineering, 2005, 16(1):74-77.

[6] Liu Zhubai, Nie Shaomin, Wang Liandong. A New Theory for Upsetting a Cylinder between Flat Platens. Chinese Journal of Mechanical Engineering, 1992, 5(4):297-303.

[7] Zhubai Liu. New Technology of Plastic Forming and its Mechanics Principle. Beijing: China Machine Press, 1995:1-52.

[8] Guohui Liu, Wenhui Xiao, Liyong Ni, Zhubai Liu. Mechanics Analysis of Cyl inder Upsetting between Flat Platen and Supporting Ring. Chinese Journal of Mechanical Engineering, 2007,18(7):854-858.

[9] Wang Liandong, Liu Zhubai. Solution of Generalized Slip-line for Upsetting a Cylinder (H/D>1) between Flat Platens. Chinese Journal of Mechanical Engineering, 1993,6(3):195-203.

[10] Liandong Wang, Zhubai Liu. Mechanical Slab Method of the Stress Field Calculation for a Cylinder(H/D>1) Being Upset. Journal of Plasticity Engineering, 1994,1(3):20-28.

[11] Yudong Yang. Control on inner crack defect of forged cold-roller of high carbon and chrome steel. Beijing: University of Science and Technology Beijing, 2007:12-18.

[12] Yong Zhou. Analysis on Forging Defects in Pipe-fitting Disc of Steel 20MnMo and Improvement of its Production Process. Special Steel Technology, 2007,13(50):41-43.

[13] Jingtao Han, Jiongjun Zhang, Zhongli Zhao et al. The Forging Process of Controiiing the Inciusion Defects for Heavy Disk Forgings and Its Appiication. Heavy Casting and Forging, 2007, (2):34-36.

[14] Meng Ren, Baoyin Zhang. RST Effect. Forging Technology,1991,16(3):5-10.

[15] Meng Ren, Baoyin Zhang, Xigang Jin. RST Effect of Large Disk-shaped Forging. Heavy Casting and Forging,1991,(4): 5-16.

[16] V Sljapic, P Hartley, I Pillinger. Observations on fracture in axi-symmetric and three-dimensional cold upsetting of brass. Journal of Materials Processing Technology, 2002, 125-126(9):267-274

[17] Takefumi Arikawa, Daisuke Yamabe, Hideki Kakimoto. Influence of Anvil Shape of Surface Crack Generation in Large Hot Forging Process. Procedia Engineering, 2014, 81: 480-485

[18] Z.J. Zhang, G.Z. Dai, S.N. Wu, L.X. Dong, L.L. Liu. Simulation of 42CrMo steel billet upsetting and its defects analyses during forming process based on the software DEFORM-3D. Materials Science and Engineering: A, 2009, 499(1-2):49-52

[19] G. Banaszek, A. Stefanik. Theoretical and laboratory modelling of the closure of metallurgical defects during forming of a forging. Journal of Materials Processing Technology, 2006, 177(1-3):238-242

[20] Y.S. Lee, S.U. Lee, C.J. Van Tyne, B.D. Joo, Y.H. Moon. Internal void closure during the forging of large cast ingots using a simulation approach. Journal of Materials Processing Technology, 2011,211(6):1136-1145

[21] G Banaszek, S Berski, H Dyja, A Kawałek. Theoretical Modelling of Metallurgical Defect Closing-Up Processes During Forming a Forging. Journal of Iron and Steel Research, International, 2013,20(9):111-116

[22] Kun Chen, Kejia Liu, Huifen Chen, Yitao Yang. A criterion for void closure in the porous model during the forging of steel ingot and its application. Computational Materials Science, 2014,91(8): 303-309

[23] Ming-Song Chen, Y.C. Lin. Numerical simulation and experimental verification of void evolution inside large forgings during 
hot working. International Journal of Plasticity, 2013,49:53-70

[24] Kun Chen, Yitao Yang, Guangjie Shao, Kejia Liu. Strain function analysis method for void closure in the forging process of the large-sized steel ingot. Computational Materials Science, 2012, 51(1):72-77

[25] Zhenshan Cui, Guangsheng Ren, Bingye Xu et al.. Numerical Simulation of Fore Closure in Solid Cylinder under Hot Forging. Journal of Plasticity Engineering,2002,9(1): 49-52.

[26] Huagui Huang. Control of High Temperature Forming Defects of Large Roller and its Numerical Simulation Study. Qinhuangdao. Yanshan University, 2006. 